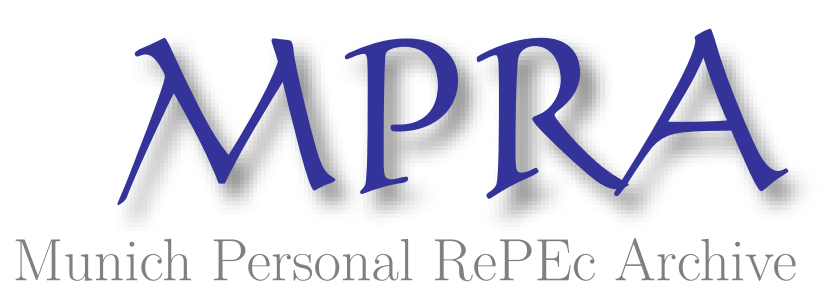

\title{
On the Effects of the BRICS on World Economic Growth
}

Bosupeng, Mpho

University of Botswana

2017

Online at https://mpra.ub.uni-muenchen.de/81757/

MPRA Paper No. 81757, posted 03 Oct 2017 14:54 UTC 


\title{
On the Effects of the BRICS on World Economic Growth
}

\author{
Author: Mpho Bosupeng \\ Email: bosupengmpho@hotmail.com
}

This is the Accepted Version!

FINAL VERSION IS HERE:

http://www.naturalspublishing.com/files/published/4f6f5krw1 cs253.pdf 


\title{
On the Effects of the BRICS on World Economic Growth
}

\begin{abstract}
The purpose of this empirical study is to examine the potential effects of the BRICS on other economies' economic growth over the period 1960-2013. This investigation deploys the Saikkonen and Lütkepohl cointegration methodology to validate long run relations between Brazil and China's economic growth and other nation's output growth. The study further uses the Toda and Yamamoto approach to Granger causality to examine long run causal links between the BRICS economic growth. The results show that all countries exhibit long run relations with China and Brazil's economic growth. In addition, the results prove that Brazil's economic growth is induced by South Africa, China and India's economic growth.
\end{abstract}

Keywords: economic growth; BRICS; developing economies; economic integration 


\section{Introduction}

The acronym BRICS refers to a set of fast developing nations namely: Brazil, Russia, India, China and South Africa. Economic growth exhibited by these economics has been quite impressive in the last decades particularly for China and India. China's economic growth has been exponential over the years. Today China is the second largest economy in the world. Additionally China's influence has been robust in matters such as exports variety, carbon dioxide emissions, global sustainable development and skills transfer in many economies. Exponential economic growth is desirable but it has shortcomings. For instance the BRICS are currently concerned with reducing emissions without hampering economic prosperity. China is currently the largest emitter of carbon dioxide globally. India also registered the highest emissions growth recently. Nonetheless, the BRICS are leading world economic growth.

The common aspect among the BRICS is that they are industrialised exporters. Economists postulated that countries such as China and India are a clear example of the export-led growth hypothesis. Export-led growth economies rely heavily on exports to drive sectors of the economy. Mineral exporting economies such as South Africa have to consider the prudent use of their resources and economic development. Over the years many economies have relied on the BRICS for their imports. Numerous studies have been examined to investigate the influence of the BRICS in matters such as inflation spillovers and market returns. Sustainable development is desirable however countries need to cooperate for this endeavour to be realised. An economy cannot be self-sufficient in all sectors.

The question is how does economic growth of the BRICS affect a given economy? How do the BRICS interact in their attempts to reach sustainable development? This paper aims to answer all these questions. This study focuses on Brazil and China to determine the long run effects of these economies' growth on other nations. The reason for focusing on China and Brazil are follows. China is currently the second largest economy and her global influence has multiplied. Brazil is also highly influential in South America. The other reason is geographical location. Brazil is located far west while China is in Asia. This is important to determine global influence of these economies without being biased to Asian economies only such as Russia, India and China. The second aim is to investigate the causal relations between members of the BRICS's economic growth patterns. The reason for this aim is that the BRICS need to depend on each other in various sectors for their own sustainable development. In this way their global influence will be prodigious (synergy).

In this paper the cointegration method proposed by Saikkonen and Lütkepohl (2000) as well as the Toda and Yamamoto (1995) causality procedure are applied. This investigation is structured as follows. Next is literature review which provides a detailed analysis of previous studies. This will be followed by data description, methodology and empirical results. Lastly a discussion and conclusion of the study follows with practical implications. An overview of the development of the BRICS shows that China grew rapidly and was followed by Brazil, India and South Africa subsequently over the period 1960-2013 (see figure 1). Note that Russia has not been included in this study due to data availability challenges. 
Figure 1: Progress of Economic Growth (1960-2013)

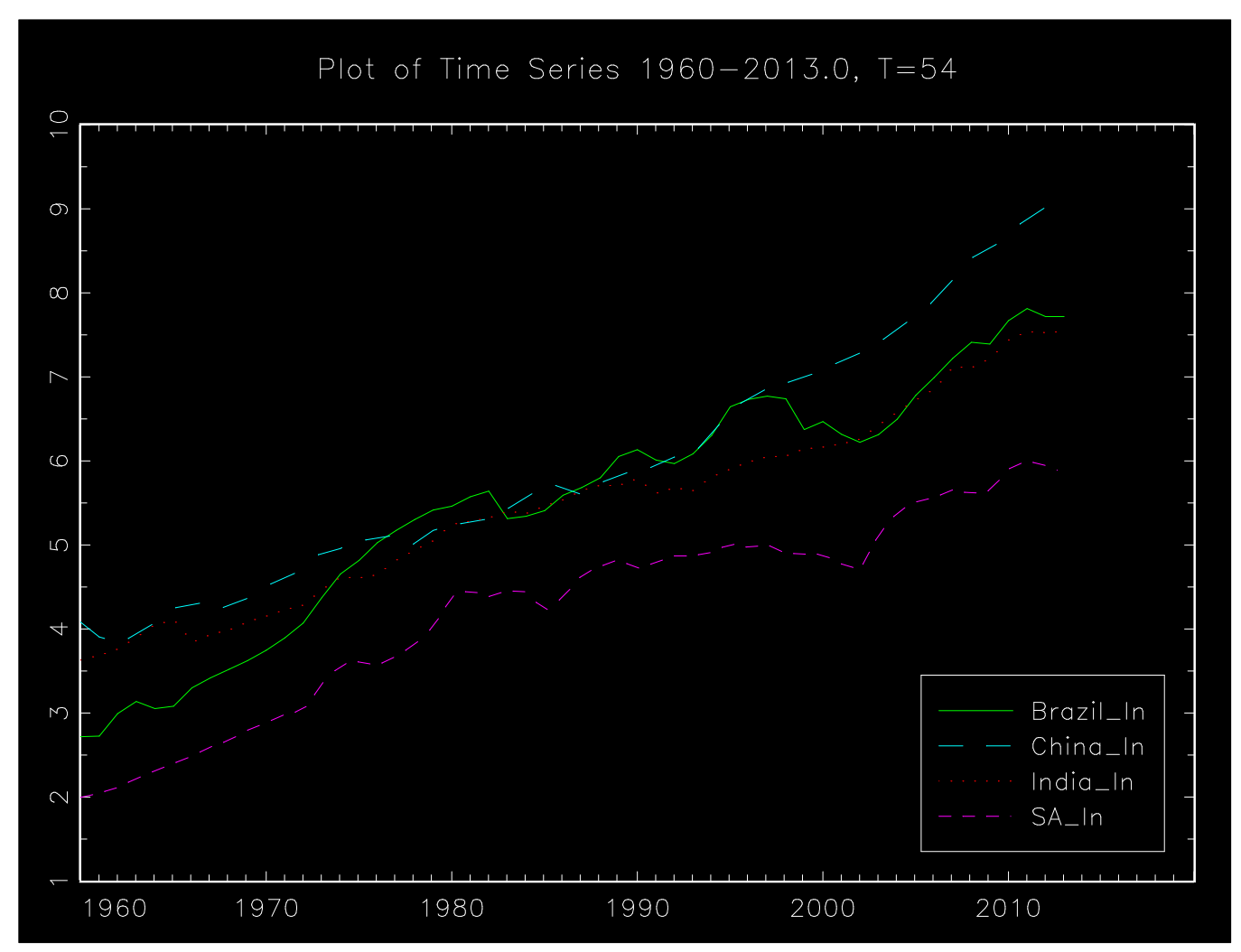

Note: The data has been converted to natural logarithms so that it is easy to monitor the volatility of the variables over the material period. Due to data unavailability Russia was not included in this analysis. Source: JMulti (4) statistical package.

\section{Literature Review}

Studies pertaining to economic growth have been numerous. Chang et al (2013) examined the effects of exports and globalization on economic growth using the corrected least square variable model for five South Caucasus countries (Azerbaijan, Armenia, Georgia, Russia and Turkey). The results of the study revealed that exports with higher energy content and globalization induce economic growth. Amador (2012) highlighted that the BRICS members Brazil, India and China possess high energy content in manufacturing exports. The authors noted that economic growth is dependent on other factors such as political and social integration. The results of this study are conceivable because political turmoil tends to disrupt economic growth in many economics especially in Africa. The problem with high energy content exports is the production of emissions in the manufacturing process. Carbon dioxide is not the only gas we should be concerned about. Sulphur dioxide is also a challenge and has detrimental effects on the environment and people. Chang et al (2013) argued that energy exports and globalisation are important determinants of economic prosperity in the South Caucasus region.

Sharma (2003) contributed by investigating the determinants of India's export performance in a simultaneous equation framework. The study examined data over the period 1970-1998 and 
the results revealed that the real appreciation of the Rupee adversely affects India's exports performance. Under this circumstance, economic growth is also affected because output depends on the positivity of net exports. As the Rupee appreciates, Indian exports become more expensive to importing economies thus registering a decline in demand. Tekin (2012) examined causation between real GDP, real exports and Foreign Domestic Investment (FDI) in least developed countries over the period 1970 to 2009. The results demonstrated that exports induce economic growth in Haiti, Rwanda and Sierra Leone. Such countries can be termed as exportled growth economies. Export led growth economies have to consider how they can reduce emissions without hampering economic growth. Economic growth was found to accelerate exports growth of the following economies: Angola, Chad and Zambia. FDI was found to Granger cause GDP in Benin and Togo.

A summary of the reviewed literature shows that economic growth is affected by several factors such as exchange rates, political and social integration. The BRICS are associated with high energy exports following (Amador, 2012). This study intends to determine the influence of the BRICS on other economies' economic growth over the period 1960 to 2013. The investigation applies the recent cointegration method proposed by Saikkonen and Lütkepohl (2000). The examination further uses the Toda and Yamamoto (1995) approach to determine the direction of causation between economic growths among the BRICS.

\section{Materials and Methods}

This study examines the relations between GDP for sixty different economies with the BRICS over the period 1960 to 2013. The data was obtained from a web source named The Global Economy (http://www.theglobaleconomy.com/). Russia was not included in this analysis due to absence of material data. Actual GDP was in billion dollars (U\$). The actual data was converted to natural logarithms before proceeding with empirical analysis. The reason is technically, it is easier to monitor the volatility of logarithmic values over the material period as compared to using raw data. It is imperative that the data set is examined for unit roots. The Augmented Dickey Fuller test (see Dickey and Fuller, 1979) was selected to test for stationarity of the variables. The testing procedure of the ADF is derived from the following generalized model:

$$
\Delta y_{t}=\alpha+\beta_{t}+\gamma y_{t-1}+\delta \Delta y_{t-1}+\cdots+\delta_{p-1} \Delta y_{t-p+1}+\varepsilon_{t},
$$

The model applied in this study is:

$$
\therefore \Delta y_{t}=\alpha+\beta_{t}+\gamma y_{t-1}+\sum_{i=1}^{k} \delta_{i} \Delta y_{t-1}+\varepsilon_{t} \text {. }
$$

The definition of terms is as follows. The regression constant is $\alpha$ and $\beta$ is the coefficient of the time trend. Following Asemota and Bala (2011) $\varepsilon_{t}$ was defined as the white noise error term. Eviews 7 was used to test the stationarity of the series. Table 1 shows the results of the Augmented Dickey Fuller test. Note that only Bangladesh series was stationary. 
Table 1: Augmented Dickey Fuller (ADF) Test Results

\begin{tabular}{|c|c|c|c|}
\hline \multirow{2}{*}{ Country } & \multicolumn{3}{|c|}{ ADF Test Statistics } \\
\hline & $1 \%$ level & $5 \%$ level & $10 \%$ level \\
\hline Brazil & $-2.402113^{1}(-4.140858)$ & $-2.402113^{2}(-3.496960)$ & $-2.402113^{3}(-3.177579)$ \\
\hline Belize & $-1.808152^{1}(-4.140858)$ & $-1.808152^{2}(-3.496960)$ & $-1.808152^{3}(-3.177579)$ \\
\hline Bolivia & $-2.511779^{1}(-4.140858)$ & $-2.511779^{2}(-3.496960)$ & $-2.511779^{3}(-3.177579)$ \\
\hline Canada & $-0.978457^{1}(-4.140858)$ & $-0.978457^{2}(-3.496960)$ & $-0.978457^{3}(-3.177579)$ \\
\hline Chile & $-3.673869^{1}(-4.140858)$ & $-3.673869(-3.496960)$ & $-3.673869(-3.177579)$ \\
\hline Colombia & $-2.428655^{1}(-4.140858)$ & $-2.428655^{2}(-3.496960)$ & $-2.428655^{3}(-3.177579)$ \\
\hline Costa Rica & $-2.528498^{1}(-4.140858)$ & $-2.528498^{2}(-3.496960)$ & $-2.528498^{3}(-3.177579)$ \\
\hline Dominica & $-2.316012^{1}(-4.140858)$ & $-2.316012^{2}(-3.496960)$ & $-2.316012^{3}(-3.177579)$ \\
\hline Ecuador & $-2.290930^{1}(-4.140858)$ & $-2.290930^{2}(-3.496960)$ & $-2.290930^{3}(-3.177579)$ \\
\hline Guatemala & $-3.553112^{1}(-4.140858)$ & $-3.553112(-3.496960)$ & $-3.553112(-3.177579)$ \\
\hline Guyana & $-0.796204^{1}(-4.140858)$ & $-0.796204^{2}(-3.496960)$ & $-0.796204^{3}(-3.177579)$ \\
\hline Honduras & $0.158081^{1}(-4.140858)$ & $0.158081^{2}(-3.496960)$ & $0.158081^{3}(-3.177579)$ \\
\hline Jamaica & $-3.165784^{1}(-4.140858)$ & $-3.165784^{2}(-3.496960)$ & $-3.165784^{3}(-3.177579)$ \\
\hline Mexico & $-2.741484^{1}(-4.140858)$ & $-2.741484^{2}(-3.496960)$ & $-2.741484^{3}(-3.177579)$ \\
\hline Nicaragua & $-3.1404822^{1}(-4.140858)$ & $-3.140482^{2}(-3.496960)$ & $-3.140482^{3}(-3.177579)$ \\
\hline Panama & $-2.084095^{1}(-4.140858)$ & $-2.084095^{2}(-3.496960)$ & $-2.084095^{3}(-3.177579)$ \\
\hline Peru & $-2.389856^{1}(-4.140858)$ & $-2.389856^{2}(-3.496960)$ & $-2.389856^{3}(-3.177579)$ \\
\hline Puerto Rico & $1.861587^{1}(-4.140858)$ & $1.861587^{2}(-3.496960)$ & $1.861587^{3}(-3.177579)$ \\
\hline Saint Vincent & $-1.559270^{1}(-4.140858)$ & $-1.559270^{2}(-3.496960)$ & $-1.559270^{3}(-3.177579)$ \\
\hline Suriname & $-1.292000^{1}(-4.140858)$ & $-1.292000^{2}(-3.496960)$ & $-1.292000^{3}(-3.177579)$ \\
\hline Trinidad \& Tobago & $-1.317052^{1}(-4.140858)$ & $-1.317052^{2}(-3.496960)$ & $-1.317052^{3}(-3.177579)$ \\
\hline USA & $1.773539^{1}(-4.140858)$ & $1.773539^{2}(-3.496960)$ & $1.773539^{3}(-3.177579)$ \\
\hline Uruguay & $-3.131048^{1}(-4.140858)$ & $-3.131048^{2}(-3.496960)$ & $-3.131048^{3}(-3.177579)$ \\
\hline Venezuela & $-1.498755^{1}(-4.140858)$ & $-1.498755^{2}(-3.496960)$ & $-1.498755^{3}(-3.177579)$ \\
\hline Algeria & $-0.989750^{1}(-4.140858)$ & $-0.989750^{2}(-3.496960)$ & $-0.989750^{3}(-3.177579)$ \\
\hline Benin & $-2.530252^{1}(-4.140858)$ & $-2.530252^{2}(-3.496960)$ & $-2.530252^{3}(-3.177579)$ \\
\hline Botswana & $-0.423076^{1}(-4.140858)$ & $-0.423076^{2}(-3.496960)$ & $-0.423076^{3}(-3.177579)$ \\
\hline Burkina Faso & $-1.629912^{1}(-4.140858)$ & $-1.629912^{2}(-3.496960)$ & $-1.629912^{3}(-3.177579)$ \\
\hline Cameroon & $-1.061231^{1}(-4.140858)$ & $-1.061231^{2}(-3.496960)$ & $-1.061231^{3}(-3.177579)$ \\
\hline Chad & $-1.534980^{1}(-4.140858)$ & $-1.534980^{2}(-3.496960)$ & $-1.534980^{3}(-3.177579)$ \\
\hline Ghana & $-0.700408^{1}(-4.140858)$ & $-0.700408^{2}(-3.496960)$ & $-0.700408^{3}(-3.177579)$ \\
\hline Ivory Coast & $-1.836463^{1}(-4.140858)$ & $-1.836463^{2}(-3.496960)$ & $-1.836463^{3}(-3.177579)$ \\
\hline Lesotho & $-2.334822^{1}(-4.140858)$ & $-2.334822^{2}(-3.496960)$ & $-2.334822^{3}(-3.177579)$ \\
\hline Liberia & $-1.903387^{1}(-4.140858)$ & $-1.903387^{2}(-3.496960)$ & $-1.903387^{3}(-3.177579)$ \\
\hline Malawi & $-2.139807^{1}(-4.140858)$ & $-2.139807^{2}(-3.496960)$ & $-2.139807^{3}(-3.177579)$ \\
\hline Madagascar & $-1.774836^{1}(-4.140858)$ & $-1.774836^{2}(-3.496960)$ & $-1.774836^{3}(-3.177579)$ \\
\hline Mauritania & $-2.015906^{1}(-4.140858)$ & $-2.015906^{2}(-3.496960)$ & $-2.015906^{3}(-3.177579)$ \\
\hline Morocco & $-1.526097^{1}(-4.140858)$ & $-1.526097^{2}(-3.496960)$ & $-1.526097^{3}(-3.177579)$ \\
\hline Niger & $-2.234723^{1}(-4.140858)$ & $-2.234723^{2}(-3.496960)$ & $-2.234723^{3}(-3.177579)$ \\
\hline Nigeria & $-1.007071^{1}(-4.140858)$ & $-1.007071^{2}(-3.496960)$ & $-1.007071^{3}(-3.177579)$ \\
\hline Congo & $-1.616850^{1}(-4.140858)$ & $-1.616850^{2}(-3.496960)$ & $-1.616850^{3}(-3.177579)$ \\
\hline Seychelles & $-1.380275^{1}(-4.140858)$ & $-1.380275^{2}(-3.496960)$ & $-1.380275^{3}(-3.177579)$ \\
\hline Sierra Leone & $-1.571073^{1}(-4.140858)$ & $-1.571073^{2}(-3.496960)$ & $-1.571073^{3}(-3.177579)$ \\
\hline South Africa & $-2.134646^{1}(-4.140858)$ & $-2.134646^{2}(-3.496960)$ & $-2.134646^{3}(-3.177579)$ \\
\hline Sudan & $-1.542140^{1}(-4.140858)$ & $-1.542140^{2}(-3.496960)$ & $-1.542140^{3}(-3.177579)$ \\
\hline Swaziland & $-1.881155^{1}(-4.140858)$ & $-1.881155^{2}(-3.496960)$ & $-1.881155^{3}(-3.177579)$ \\
\hline Togo & $-1.193167^{1}(-4.140858)$ & $-1.193167^{2}(-3.496960)$ & $-1.193167^{3}(-3.177579)$ \\
\hline Uganda & $-3.420546^{1}(-4.140858)$ & $-3.420546^{2}(-3.496960)$ & $-3.420546(-3.177579)$ \\
\hline Zambia & $-1.032484^{1}(-4.140858)$ & $-1.032484^{2}(-3.496960)$ & $-1.032484^{3}(-3.177579)$ \\
\hline Zimbabwe & $-1.829366^{1}(-4.140858)$ & $-1.829366^{2}(-3.496960)$ & $-1.829366^{3}(-3.177579)$ \\
\hline Bangladesh & $-5.177530(-4.140858)$ & $-5.177530(-3.496960)$ & $-5.177530(-3.177579)$ \\
\hline China & $-0.804449^{1}(-4.140858)$ & $-0.804449^{2}(-3.496960)$ & $-0.804449^{3}(-3.177579)$ \\
\hline
\end{tabular}




\begin{tabular}{llll} 
Hong Kong & $-0.593099^{1}(-4.140858)$ & $-0.593099^{2}(-3.496960)$ & $-0.593099^{3}(-3.177579)$ \\
India & $-1.686225^{1}(-4.140858)$ & $-1.686225^{2}(-3.496960)$ & $-1.686225^{3}(-3.177579)$ \\
Israel & $-1.830077^{1}(-4.140858)$ & $-1.830077^{2}(-3.496960)$ & $-1.830077^{3}(-3.177579)$ \\
Japan & $-0.125997^{1}(-4.140858)$ & $-0.125997^{2}(-3.496960)$ & $-0.125997^{3}(-3.177579)$ \\
Malaysia & $-1.651926^{1}(-4.140858)$ & $-1.651926^{2}(-3.496960)$ & $-1.651926^{3}(-3.177579)$ \\
Nepal & $-2.366434^{1}(-4.140858)$ & $-2.366434^{2}(-3.496960)$ & $-2.366434^{3}(-3.177579)$ \\
Oman & $-1.219531^{1}(-4.140858)$ & $-1.219531^{2}(-3.496960)$ & $-1.219531^{3}(-3.177579)$ \\
Pakistan & $-2.469553^{1}(-4.140858)$ & $-2.469553^{2}(-3.496960)$ & $-2.469553^{3}(-3.177579)$ \\
\hline
\end{tabular}

The ADF test statistics are reported above. The critical values are as follows: $-[4.140858]$ is the critical value at $1 \%$ level; -[3.496960] is the critical value at 5\% level and -[3.177579] is the critical value at $10 \%$ level. The numbers in brackets are critical values. Superscripts 1, 2, 3 indicate statistical significance at 1\%, 5\%, and 10\% critical levels. The results are based on the model: $\Delta y_{t}=\alpha+\beta_{t}+\gamma y_{t-1}+\sum_{i=1}^{k} \delta_{i} \Delta y_{t-1}+\varepsilon_{t}$. Eviews 7 was used to compute the ADF unit root test. The null hypothesis for the test is "series $\mathrm{x}$, has a unit root".

\section{Saikkonen and Lütkepohl (2000) Cointegration Model}

In this study, it is important to examine the long run relations between the series. This paper applies the recent cointegration method proposed by Saikkonen and Lütkepohl (2000). Cointegrated variables will be attracted to each other therefore resulting in long run affiliations. Even though the Johansen cointegration test and the Saikkonen and Lütkepohl test are almost similar, there are technical differences. Firstly, the Saikkonen and Lütkepohl test is different technically because it estimates the deterministic term first and then subtracts it from the time series observations unlike the Johansen method. Saikkonen and Lütkepohl (2000) commenced their model by considering a $\operatorname{VAR}(p)$ process of the form:

$$
y_{t}=v+A_{1} y_{t-1}+\cdots+A_{p} y_{t-p}+\varepsilon_{t} \quad t=p+1, p+2, \ldots,
$$

Following Saikkonen and Lütkepohl (2000) allow $A_{J}$ to be $n \times n$ coefficient matrices while $\varepsilon_{t}$ is an $n \times 1$ is a stochastic error term assumed to be a martingale difference sequence with $E$ $\left(\varepsilon_{t} \mid \varepsilon_{s,} s<t\right)=0$. The non-stochastic positive definite conditional covariance matrix was defined as $E\left(\varepsilon_{t} \dot{\varepsilon}_{t} \mid \varepsilon_{S}, S<t\right)=\Omega$. The resulting final error correction model formed by subtracting $y_{t-1}$ on both sides of the $\operatorname{VAR}(p)$ above is

$$
\Delta \tilde{y}_{t}=v+\Pi \tilde{y}_{t-1}+\sum_{j=1}^{p-1} \Gamma_{j} \Delta \tilde{y}_{t-j}+\varepsilon_{t} \quad t=p+1, p+2, \ldots,
$$

The definition of terms is $\Pi=-\left(I_{n}-A_{1}-\cdots-A_{p}\right)$ while $\Gamma_{j}=-\left(A_{j+1}+\cdots+A_{p}\right)$ $(j=1, \ldots, p-1)$. The test validates if $H\left(r_{0}\right): r k(\Pi)=r_{0}$. 


\section{The Toda and Yamamoto (1995) Approach to Granger Causality}

The aim of this paper is to investigate the long run causation between income series. However, in this study the other challenge is determining the direction of causal affiliations between income series of the BRICS. The Toda and Yamamoto (1995) approach is the most suitable because it does not require pre-tests for cointegration. The Granger causality test (see Granger, 1969) was not selected because not all data in this study is non-stationary (Bangladesh income series was stationary). The Toda and Yamamoto (1995) technique can apply even if the series does not have unit roots. Granger causality also has several limitations. Originally, if the variables under consideration are driven by a common third process with different lags, there is a possibility of failing to reject the alternative hypothesis of Granger causality. In addition, Granger causality is often based on the assumption that causal relations are a result of cointegration. The advantage of the Toda and Yamamoto (1995) approach is that the VAR's formulated in the levels can be estimated even if the processes may be integrated or cointegrated of an arbitrary order. Wolde-Rufael (2005) observed that the Toda and Yamamoto (1995) approach fits a standard vector autoregressive model in the levels of the variables. In consequence, this minimizes risks associated with the likelihood of wrongly identifying the order of integration of the series (Mavrotas and Kelly, 2001).

The literature has developed a number of cointegration methods following the contributions of Saikkonen and Lütkepohl (2000); Johansen and Juselius (1990); Johansen (1988b, 1991a); Granger (1981); Granger and Weiss (1983); Engle and Granger (1987); Granger and Engle (1985); Stock (1987); Phillips and Durlauf (1986); Phillips and Park (1986); Phillips and Ouilaris (1986); Stock and Watson (1987); Park (1992a, 1990b); Phillips and Hansen (1990); Hovarth and Watson (1995); Saikkonen (1992) and Elliot (1998). Toda and Yamamoto (1995) noted that if economic variables are not cointegrated then the VAR should be estimated in firstorder differences of the variables to validate the conventional asymptotic theory. In consequence, the Toda and Yamamoto (1995) approach is applicable even if the VAR may be stationary, integrated of an arbitrary order or cointegrated of an arbitrary order.

This study applies the Toda and Yamamoto (1995) approach as discussed by Wolde-Rufael (2005). The testing procedure starts by augmenting the correct VAR order $k$ by the maximal order of integration $d_{\max }$ (Wolde-Rufael, 2005). Following this, a $\left(k+d_{\text {max }}\right)^{\text {th }}$ order of the VAR is estimated and the coefficients of the last lagged $d_{\max }$ vector are ignored (Caporale and Pittis, 1999; Rambaldi and Doran, 1996; Rambaldi, 1997; Zapata and Rambaldi, 1997). Denote two income series as LX and LY. The VAR system of the variables can now be shown as:

$$
\begin{gathered}
L X_{t}=\alpha_{0}+\sum_{i=1}^{k} \alpha_{1 i} L X_{t-i}+\sum_{j=k+1}^{d_{\max }} \alpha_{2 j} L X_{t-j}+\sum_{i=1}^{k} \emptyset_{1 i} L Y_{t-i}+\sum_{j=k+1}^{d_{\max }} \emptyset_{2 j} L Y_{t-j}+\lambda_{1 t} \\
L Y_{t}=\beta_{0}+\sum_{i=1}^{k} \beta_{1 i} L Y_{t-1}+\sum_{j=k+1}^{d_{\max }} \beta_{2 j} L Y_{t-j}+\sum_{i=1}^{k} \delta_{1 i} L X_{t-i}+\sum_{j=k+1}^{d_{\max }} \delta_{2 j} L X_{t-j}+\lambda_{2 t}
\end{gathered}
$$




\section{Empirical Results}

The Saikkonen and Lükepohl test was carried out at 90\%, 95\% and 99\% critical levels using JMulti (4) statistical package. The results show that there is a long run relationship between all the countries' economic growth and the two countries income (Brazil and China). Tables 2 and 3 represent the results of the cointegration test. Note that $\rho$-values less than the critical levels of $90 \%, 95 \%$ and $99 \%$ represent cointegration.

Table 2: Results of the Saikkonen and Lütkepohl Cointegration Test (Brazil)

\begin{tabular}{|c|c|c|c|c|c|c|c|c|c|c|c|c|}
\hline Country & $\mathbf{r}_{0}$ & LR & $90 \%$ & $95 \%$ & $99 \%$ & $\rho$-value & $\mathbf{r}_{0}$ & LR & $90 \%$ & $95 \%$ & $99 \%$ & $\rho$-value \\
\hline Belize & 0 & 6.4300 & 13.880 & 15.760 & 19.710 & $0.72400^{1,2,3}$ & 1 & 1.0200 & 5.470 & 6.790 & 9.730 & $0.78630^{1,2,3}$ \\
\hline Bolivia & 0 & 5.7100 & 13.880 & 15.760 & 19.710 & $0.80480^{1,2,3}$ & 1 & 2.4600 & 5.470 & 6.790 & 9.730 & $0.43960^{1,2,3}$ \\
\hline Canada & 0 & 9.5800 & 13.880 & 15.760 & 19.710 & $0.37820^{1,2,3}$ & 1 & 1.1600 & 5.470 & 6.790 & 9.730 & $0.74950^{1,2,3}$ \\
\hline Chile & 0 & 13.7900 & 13.880 & 15.760 & 19.710 & $0.10320^{1,2,3}$ & 1 & 1.7400 & 5.470 & 6.790 & 9.730 & $0.59920^{1,2,3}$ \\
\hline Colombia & 0 & 9.6900 & 13.880 & 15.760 & 19.710 & $0.36730^{1,2,3}$ & 1 & 2.1800 & 5.470 & 6.790 & 9.730 & $0.49880^{1,2,3}$ \\
\hline Costa Rica & 0 & 12.5200 & 13.880 & 15.760 & 19.710 & $0.15880^{1,2,3}$ & 1 & 2.4500 & 5.470 & 6.790 & 9.730 & $0.44130^{1,2,3}$ \\
\hline Dominica & 0 & 12.5300 & 13.880 & 15.760 & 19.710 & $0.15810^{1,2,3}$ & 1 & 1.9300 & 5.470 & 6.790 & 9.730 & $0.55330^{1,2,3}$ \\
\hline Ecuador & 0 & 11.7100 & 13.880 & 15.760 & 19.710 & $0.20560^{1,2,3}$ & 1 & 2.1100 & 5.470 & 6.790 & 9.730 & $0.51280^{1,2,3}$ \\
\hline Guatemala & 0 & 9.6700 & 13.880 & 15.760 & 19.710 & $0.36960^{1,2,3}$ & 1 & 1.9300 & 5.470 & 6.790 & 9.730 & $0.55370^{1,2,3}$ \\
\hline Guyana & 0 & 7.2800 & 13.880 & 15.760 & 19.710 & $0.63000^{1,2,3}$ & 1 & 0.5600 & 5.470 & 6.790 & 9.730 & $0.90580^{2,3}$ \\
\hline Honduras & 0 & 11.0400 & 13.880 & 15.760 & 19.710 & $0.25240^{1,2,3}$ & 1 & 1.6400 & 5.470 & 6.790 & 9.730 & $0.62440^{1,2,3}$ \\
\hline Jamaica & 0 & 8.8800 & 13.880 & 15.760 & 19.710 & $0.44970^{1,2,3}$ & 1 & 2.1100 & 5.470 & 6.790 & 9.730 & $0.51210^{1,2,3}$ \\
\hline Mexico & 0 & 14.7400 & 13.880 & 15.760 & 19.710 & $0.07330^{1,2,3}$ & 1 & 2.6500 & 5.470 & 6.790 & 9.730 & $0.40310^{1,2,3}$ \\
\hline Nicaragua & 0 & 11.4900 & 13.880 & 15.760 & 19.710 & $0.22050^{1,2,3}$ & 1 & 2.3100 & 5.470 & 6.790 & 9.730 & $0.46940^{1,2,3}$ \\
\hline Panama & 0 & 7.0600 & 13.880 & 15.760 & 19.710 & $0.6560^{1,2,3}$ & 1 & 2.2500 & 5.470 & 6.790 & 9.730 & $0.48350^{1,2,3}$ \\
\hline Peru & 0 & 7.9600 & 13.880 & 15.760 & 19.710 & $0.55130^{1,2,3}$ & 1 & 1.3900 & 5.470 & 6.790 & 9.730 & $0.68920^{1,2,3}$ \\
\hline Puerto Rico & 0 & 7.8200 & 13.880 & 15.760 & 19.710 & $0.56830^{1,2,3}$ & 1 & 0.1400 & 5.470 & 6.790 & 9.730 & $0.98890^{3}$ \\
\hline Saint Lucia & 0 & 8.5400 & 13.880 & 15.760 & 19.710 & $0.48620^{1,2,3}$ & 1 & 0.9300 & 5.470 & 6.790 & 9.730 & $0.81230^{1,2,3}$ \\
\hline Suriname & 0 & 6.0700 & 13.880 & 15.760 & 19.710 & $0.76730^{1,2,3}$ & 1 & 1.9000 & 5.470 & 6.790 & 9.730 & $0.56040^{1,2,3}$ \\
\hline T \& Tobago & 0 & 7.3500 & 13.880 & 15.760 & 19.710 & $0.62200^{1,2,3}$ & 1 & 2.1000 & 5.470 & 6.790 & 9.730 & $0.51630^{1,2,3}$ \\
\hline USA & 0 & 17.0800 & 13.880 & 15.760 & 19.710 & $0.02980^{1,2,3}$ & 1 & 1.7800 & 5.470 & 6.790 & 9.730 & $0.59080^{1,2,3}$ \\
\hline Uruguay & 0 & 13.8200 & 13.880 & 15.760 & 19.710 & $0.10210^{1,2,3}$ & 1 & 2.6200 & 5.470 & 6.790 & 9.730 & $0.40970^{1,2,3}$ \\
\hline Venezuela & 0 & 5.0400 & 13.880 & 15.760 & 19.710 & $0.86730^{1,2,3}$ & 1 & 2.6200 & 5.470 & 6.790 & 9.730 & $0.40940^{1,2,3}$ \\
\hline Algeria & 0 & 6.9300 & 13.880 & 15.760 & 19.710 & $0.67160^{1,2,3}$ & 1 & 1.4800 & 5.470 & 6.790 & 9.730 & $0.66570^{1,2,3}$ \\
\hline Benin & 0 & 7.7200 & 13.880 & 15.760 & 19.710 & $0.57920^{1,2,3}$ & 1 & 3.7300 & 5.470 & 6.790 & 9.730 & $0.24260^{1,2,3}$ \\
\hline Botswana & 0 & 9.0800 & 13.880 & 15.760 & 19.710 & $0.42830^{1,2,3}$ & 1 & 0.7100 & 5.470 & 6.790 & 9.730 & $0.87010^{1,2,3}$ \\
\hline Burkina Faso & 0 & 7.5000 & 13.880 & 15.760 & 19.710 & $0.60500^{1,2,3}$ & 1 & 2.8300 & 5.470 & 6.790 & 9.730 & $0.37240^{1,2,3}$ \\
\hline Burundi & 0 & 4.3300 & 13.880 & 15.760 & 19.710 & $0.92090^{2,3}$ & 1 & 1.8200 & 5.470 & 6.790 & 9.730 & $0.57940^{1,2,3}$ \\
\hline Cameroon & 0 & 9.5800 & 13.880 & 15.760 & 19.710 & $0.37820^{1,2,3}$ & 1 & 1.1600 & 5.470 & 6.790 & 9.730 & $0.74950^{1,2,3}$ \\
\hline Chad & 0 & 14.9600 & 13.880 & 15.760 & 19.710 & $0.06760^{1,2,3}$ & 1 & 1.6600 & 5.470 & 6.790 & 9.730 & $0.61850^{1,2,3}$ \\
\hline Ghana & 0 & 8.8400 & 13.880 & 15.760 & 19.710 & $0.45370^{1,2,3}$ & 1 & 0.8300 & 5.470 & 6.790 & 9.730 & $0.83750^{1,2,3}$ \\
\hline Ivory Coast & 0 & 15.7600 & 13.880 & 15.760 & 19.710 & $0.04990^{1,2,3}$ & 1 & 1.1900 & 5.470 & 6.790 & 9.730 & $0.74250^{1,2,3}$ \\
\hline Lesotho & 0 & 16.5100 & 13.880 & 15.760 & 19.710 & $0.03740^{1,2,3}$ & 1 & 2.7300 & 5.470 & 6.790 & 9.730 & $0.39000^{1,2,3}$ \\
\hline Liberia & 0 & 5.8100 & 13.880 & 15.760 & 19.710 & $0.79480^{1,2,3}$ & 1 & 2.1800 & 5.470 & 6.790 & 9.730 & $0.49750^{1,2,3}$ \\
\hline Malawi & 0 & 12.0000 & 13.880 & 15.760 & 19.710 & $0.18810^{1,2,3}$ & 1 & 1.9500 & 5.470 & 6.790 & 9.730 & $0.54910^{1,2,3}$ \\
\hline Madagascar & 0 & 6.4600 & 13.880 & 15.760 & 19.710 & $0.72400^{1,2,3}$ & 1 & 2.0000 & 5.470 & 6.790 & 9.730 & $0.53830^{1,2,3}$ \\
\hline Mauritania & 0 & 16.4700 & 13.880 & 15.760 & 19.710 & $0.03800^{1,2,3}$ & 1 & 2.9500 & 5.470 & 6.790 & 9.730 & $0.35140^{1,2,3}$ \\
\hline Morocco & 0 & 12.9200 & 13.880 & 15.760 & 19.710 & $0.13890^{1,2,3}$ & 1 & 2.4600 & 5.470 & 6.790 & 9.730 & $0.43920^{1,2,3}$ \\
\hline Niger & 0 & 7.8400 & 13.880 & 15.760 & 19.710 & $0.56590^{1,2,3}$ & 1 & 2.4900 & 5.470 & 6.790 & 9.730 & $0.43460^{1,2,3}$ \\
\hline Nigeria & 0 & 5.3400 & 13.880 & 15.760 & 19.710 & $0.84060^{1,2,3}$ & 1 & 0.9500 & 5.470 & 6.790 & 9.730 & $0.80640^{1,2,3}$ \\
\hline Congo & 0 & 7.0500 & 13.880 & 15.760 & 19.710 & $0.65760^{1,2,3}$ & 1 & 2.3900 & 5.470 & 6.790 & 9.730 & $0.45340^{1,2,3}$ \\
\hline Seychelles & 0 & 10.8500 & 13.880 & 15.760 & 19.710 & $0.26670^{1,2,3}$ & 1 & 1.3300 & 5.470 & 6.790 & 9.730 & $0.70330^{1,2,3}$ \\
\hline Sierra Leone & 0 & 6.6200 & 13.880 & 15.760 & 19.710 & $0.70690^{1,2,3}$ & 1 & 1.9400 & 5.470 & 6.790 & 9.730 & $0.55260^{1,2,3}$ \\
\hline
\end{tabular}




\begin{tabular}{lllllllllllll} 
South Africa & 0 & 13.1100 & 13.880 & 15.760 & 19.710 & $0.13030^{1,2,3}$ & 1 & 1.7300 & 5.470 & 6.790 & 9.730 & $0.60280^{1,2,3}$ \\
Sudan & 0 & 6.0600 & 13.880 & 15.760 & 19.710 & $0.76770^{1,2,3}$ & 1 & 2.5200 & 5.470 & 6.790 & 9.730 & $0.42890^{1,2,3}$ \\
Swaziland & 0 & 10.8900 & 13.880 & 15.760 & 19.710 & $0.26340^{1,2,3}$ & 1 & 1.8400 & 5.470 & 6.790 & 9.730 & $0.57600^{1,2,3}$ \\
Togo & 0 & 16.2100 & 13.880 & 15.760 & 19.710 & $0.04210^{1,2,3}$ & 1 & 2.7100 & 5.470 & 6.790 & 9.730 & $0.39380^{1,2,3}$ \\
Uganda & 0 & 13.5300 & 13.880 & 15.760 & 19.710 & $0.11300^{1,2,3}$ & 1 & 2.4000 & 5.470 & 6.790 & 9.730 & $0.45230^{1,2,3}$ \\
Zambia & 0 & 8.5800 & 13.880 & 15.760 & 19.710 & $0.48160^{1,2,3}$ & 1 & 0.9600 & 5.470 & 6.790 & 9.730 & $0.80180^{1,2,3}$ \\
Zimbabwe & 0 & 10.3600 & 13.880 & 15.760 & 19.710 & $0.30650^{1,2,3}$ & 1 & 2.4400 & 5.470 & 6.790 & 9.730 & $0.44440^{1,2,3}$ \\
Bangladesh & 0 & 15.0900 & 13.880 & 15.760 & 19.710 & $0.06430^{1,2,3}$ & 1 & 2.3200 & 5.470 & 6.790 & 9.730 & $0.46850^{1,2,3}$ \\
China & 0 & 8.7100 & 13.880 & 15.760 & 19.710 & $0.46820^{1,2,3}$ & 1 & 0.0800 & 5.470 & 6.790 & 9.730 & 0.99540 \\
Hong Kong & 0 & 13.6300 & 13.880 & 15.760 & 19.710 & $0.10920^{1,2,3}$ & 1 & 1.1700 & 5.470 & 6.790 & 9.730 & $0.74560^{1,2,3}$ \\
India & 0 & 7.3600 & 13.880 & 15.760 & 19.710 & $0.62130^{1,2,3}$ & 1 & 1.2300 & 5.470 & 6.790 & 9.730 & $0.73040^{1,2,3}$ \\
Israel & 0 & 8.3500 & 13.880 & 15.760 & 19.710 & $0.50790^{1,2,3}$ & 1 & 1.1300 & 5.470 & 6.790 & 9.730 & $0.75690^{1,2,3}$ \\
Japan & 0 & 8.1500 & 13.880 & 15.760 & 19.710 & $0.52960^{1,2,3}$ & 1 & 0.3400 & 5.470 & 6.790 & 9.730 & $0.95630^{3}$ \\
Malaysia & 0 & 11.3400 & 13.880 & 15.760 & 19.710 & $0.23080^{1,2,3}$ & 1 & 1.3200 & 5.470 & 6.790 & 9.730 & $0.70730^{1,2,3}$ \\
Nepal & 0 & 7.6500 & 13.880 & 15.760 & 19.710 & $0.58720^{1,2,3}$ & 1 & 2.3100 & 5.470 & 6.790 & 9.730 & $0.47130^{1,2,3}$ \\
Oman & 0 & 12.7800 & 13.880 & 15.760 & 19.710 & $0.14570^{1,2,3}$ & 1 & 0.7700 & 5.470 & 6.790 & 9.730 & $0.85270^{1,2,3}$ \\
Pakistan & 0 & 13.2300 & 13.880 & 15.760 & 19.710 & $0.12500^{1,2,3}$ & 1 & 2.6600 & 5.470 & 6.790 & 9.730 & $0.40140^{1,2,3}$ \\
\hline
\end{tabular}

Note: ${ }^{1}$ shows statistical significance at $90 \%$ critical level; ${ }^{2}$ shows statistical significance at $95 \%$ critical level $;{ }^{3}$ shows statistical significance at $99 \%$ critical level. Note that $\rho$-values less than critical levels of $90 \%, 95 \%$ and $99 \%$ represent cointegration. The test was carried out using JMulti 4 statistical package. The deterministic term of the VECM was defined as $D_{t}=u_{o}+u_{1 t}$. Superscripts 1, 2, 3 show statistical significance at $90 \%, 95 \%$, and $99 \%$ critical levels. LR = Likelihood Ratio. Superscripts 1 , 2,3 show statistical significance at $90 \%, 95 \%$, and $99 \%$ critical levels.

Table 3: Results of the Saikkonen and Lütkepohl Cointegration Test (China)

\begin{tabular}{|c|c|c|c|c|c|c|c|c|c|c|c|c|}
\hline Country & $\mathbf{r}_{0}$ & LR & $90 \%$ & $95 \%$ & $99 \%$ & $\rho$-value & $\mathbf{r}_{0}$ & LR & $90 \%$ & $95 \%$ & $99 \%$ & $\rho$-value \\
\hline Brazil & 0 & 8.7100 & 13.880 & 15.760 & 19.710 & $0.46820^{1,2,3}$ & 1 & 0.0800 & 5.470 & 6.790 & 9.730 & 0.99540 \\
\hline Belize & 0 & 8.7500 & 13.880 & 15.760 & 19.710 & $0.46310^{1,2,3}$ & 1 & 0.2700 & 5.470 & 6.790 & 9.730 & $0.96830^{3}$ \\
\hline Bolivia & 0 & 10.0500 & 13.880 & 15.760 & 19.710 & $0.33340^{1,2,3}$ & 1 & 0.8700 & 5.470 & 6.790 & 9.730 & $0.82600^{1,2,3}$ \\
\hline Canada & 0 & 6.0400 & 13.880 & 15.760 & 19.710 & $0.77030^{1,2,3}$ & 1 & 0.3300 & 5.470 & 6.790 & 9.730 & $0.95720^{3}$ \\
\hline Chile & 0 & 17.4500 & 13.880 & 15.760 & 19.710 & $0.02560^{1,2,3}$ & 1 & 0.6600 & 5.470 & 6.790 & 9.730 & $0.88270^{1,2,3}$ \\
\hline Colombia & 0 & 6.6400 & 13.880 & 15.760 & 19.710 & $0.70390^{1,2,3}$ & 1 & 0.2200 & 5.470 & 6.790 & 9.730 & $0.97680^{3}$ \\
\hline Costa Rica & 0 & 9.1900 & 13.880 & 15.760 & 19.710 & $0.41730^{1,2,3}$ & 1 & 0.2300 & 5.470 & 6.790 & 9.730 & $0.97500^{3}$ \\
\hline Dominica & 0 & 5.6700 & 13.880 & 15.760 & 19.710 & $0.80870^{1,2,3}$ & 1 & 0.4900 & 5.470 & 6.790 & 9.730 & $0.92270^{2,3}$ \\
\hline Ecuador & 0 & 7.9100 & 13.880 & 15.760 & 19.710 & $0.55740^{1,2,3}$ & 1 & 1.3400 & 5.470 & 6.790 & 9.730 & $0.70160^{1,2,3}$ \\
\hline Guatemala & 0 & 5.7300 & 13.880 & 15.760 & 19.710 & $0.80270^{1,2,3}$ & 1 & 0.0100 & 5.470 & 6.790 & 9.730 & 0.99980 \\
\hline Guyana & 0 & 5.7100 & 13.880 & 15.760 & 19.710 & $0.80490^{1,2,3}$ & 1 & 1.1300 & 5.470 & 6.790 & 9.730 & $0.75670^{1,2,3}$ \\
\hline Honduras & 0 & 6.2600 & 13.880 & 15.760 & 19.710 & $0.74630^{1,2,3}$ & 1 & 0.0100 & 5.470 & 6.790 & 9.730 & 0.99990 \\
\hline Jamaica & 0 & 9.0100 & 13.880 & 15.760 & 19.710 & $0.43570^{1,2,3}$ & 1 & 0.6300 & 5.470 & 6.790 & 9.730 & $0.88800^{1,2,3}$ \\
\hline Mexico & 0 & 12.9700 & 13.880 & 15.760 & 19.710 & $0.13690^{1,2,3}$ & 1 & 0.5300 & 5.470 & 6.790 & 9.730 & $0.91320^{2,3}$ \\
\hline Nicaragua & 0 & 9.9600 & 13.880 & 15.760 & 19.710 & $0.34190^{1,2,3}$ & 1 & 0.4500 & 5.470 & 6.790 & 9.730 & $0.93210^{2,3}$ \\
\hline Panama & 0 & 7.8900 & 13.880 & 15.760 & 19.710 & $0.55960^{1,2,3}$ & 1 & 0.2600 & 5.470 & 6.790 & 9.730 & $0.97120^{3}$ \\
\hline Peru & 0 & 5.9400 & 13.880 & 15.760 & 19.710 & $0.78110^{1,2,3}$ & 1 & 0.1500 & 5.470 & 6.790 & 9.730 & $0.98810^{3}$ \\
\hline Puerto Rico & 0 & 9.1900 & 13.880 & 15.760 & 19.710 & $0.41640^{1,2,3}$ & 1 & 0.4200 & 5.470 & 6.790 & 9.730 & $0.93930^{2,3}$ \\
\hline Saint Lucia & 0 & 12.4400 & 13.880 & 15.760 & 19.710 & $0.16310^{1,2,3}$ & 1 & 0.3600 & 5.470 & 6.790 & 9.730 & $0.95130^{3}$ \\
\hline Suriname & 0 & 6.8900 & 13.880 & 15.760 & 19.710 & $0.67530^{1,2,3}$ & 1 & 1.1300 & 5.470 & 6.790 & 9.730 & $0.75760^{1,2,3}$ \\
\hline T \& Tobago & 0 & 4.5800 & 13.880 & 15.760 & 19.710 & $0.90360^{2,3}$ & 1 & 0.1800 & 5.470 & 6.790 & 9.730 & $0.98280^{3}$ \\
\hline USA & 0 & 5.9400 & 13.880 & 15.760 & 19.710 & $0.78100^{1,2,3}$ & 1 & 3.3600 & 5.470 & 6.790 & 9.730 & $0.28940^{1,2,3}$ \\
\hline Uruguay & 0 & 10.4600 & 13.880 & 15.760 & 19.710 & $0.29810^{1,2,3}$ & 1 & 0.6100 & 5.470 & 6.790 & 9.730 & $0.89350^{1,2,3}$ \\
\hline Venezuela & 0 & 5.8600 & 13.880 & 15.760 & 19.710 & $0.78930^{1,2,3}$ & 1 & 1.4000 & 5.470 & 6.790 & 9.730 & $0.68490^{1,2,3}$ \\
\hline Algeria & 0 & 4.5000 & 13.880 & 15.760 & 19.710 & $0.75270^{1,2,3}$ & 1 & 0.3400 & 5.470 & 6.790 & 9.730 & $0.95630^{3}$ \\
\hline Benin & 0 & 6.2000 & 13.880 & 15.760 & 19.710 & $0.84030^{1,2,3}$ & 1 & 0.5300 & 5.470 & 6.790 & 9.730 & $0.91330^{1,2,3}$ \\
\hline Botswana & 0 & 5.3400 & 13.880 & 15.760 & 19.710 & $0.57380^{1,2,3}$ & 1 & 0.9300 & 5.470 & 6.790 & 9.730 & $0.81080^{1,2,3}$ \\
\hline Burkina Faso & 0 & 7.7700 & 13.880 & 15.760 & 19.710 & $0.87700^{1,2,3}$ & 1 & 1.0600 & 5.470 & 6.790 & 9.730 & $0.77670^{1,2,3}$ \\
\hline Burundi & 0 & 5.3400 & 13.880 & 15.760 & 19.710 & $0.69800^{1,2,3}$ & 1 & 1.9100 & 5.470 & 6.790 & 9.730 & $0.55780^{1,2,3}$ \\
\hline
\end{tabular}




\begin{tabular}{|c|c|c|c|c|c|c|c|c|c|c|c|c|}
\hline Cameroon & 0 & 4.9200 & 13.880 & 15.760 & 19.710 & $0.74050^{1,2,3}$ & 1 & 0.5900 & 5.470 & 6.790 & 9.730 & $0.89980^{1,2,3}$ \\
\hline Chad & 0 & 6.6900 & 13.880 & 15.760 & 19.710 & $0.69270^{1,2,3}$ & 1 & 0.5700 & 5.470 & 6.790 & 9.730 & $0.90390^{2,3}$ \\
\hline Ghana & 0 & 6.3200 & 13.880 & 15.760 & 19.710 & $0.74050^{1,2,3}$ & 1 & 0.6500 & 5.470 & 6.790 & 9.730 & $0.88340^{1,2,3}$ \\
\hline Ivory Coast & 0 & 6.7400 & 13.880 & 15.760 & 19.710 & $0.69270^{1,2,3}$ & 1 & 1.8400 & 5.470 & 6.790 & 9.730 & $0.57630^{1,2,3}$ \\
\hline Lesotho & 0 & 7.5400 & 13.880 & 15.760 & 19.710 & $0.60070^{1,2,3}$ & 1 & 0.5600 & 5.470 & 6.790 & 9.730 & $0.90740^{2,3}$ \\
\hline Liberia & 0 & 11.0600 & 13.880 & 15.760 & 19.710 & $0.25040^{1,2,3}$ & 1 & 0.7000 & 5.470 & 6.790 & 9.730 & $0.87090^{1,2,3}$ \\
\hline Malawi & 0 & 9.0400 & 13.880 & 15.760 & 19.710 & $0.43250^{1,2,3}$ & 1 & 0.4100 & 5.470 & 6.790 & 9.730 & $0.94000^{2,3}$ \\
\hline Madagascar & 0 & 5.0100 & 13.880 & 15.760 & 19.710 & $0.86940^{1,2,3}$ & 1 & 2.0600 & 5.470 & 6.790 & 9.730 & $0.52490^{1,2,3}$ \\
\hline Mauritania & 0 & 6.2500 & 13.880 & 15.760 & 19.710 & $0.74770^{1,2,3}$ & 1 & 1.1000 & 5.470 & 6.790 & 9.730 & $0.76630^{1,2,3}$ \\
\hline Morocco & 0 & 6.7800 & 13.880 & 15.760 & 19.710 & $0.68870^{1,2,3}$ & 1 & 0.0600 & 5.470 & 6.790 & 9.730 & 0.99710 \\
\hline Niger & 0 & 7.9200 & 13.880 & 15.760 & 19.710 & $0.55670^{1,2,3}$ & 1 & 0.6300 & 5.470 & 6.790 & 9.730 & $0.88870^{1,2,3}$ \\
\hline Nigeria & 0 & 6.4900 & 13.880 & 15.760 & 19.710 & $0.72170^{1,2,3}$ & 1 & 1.5800 & 5.470 & 6.790 & 9.730 & $0.64020^{1,2,3}$ \\
\hline Congo & 0 & 8.2200 & 13.880 & 15.760 & 19.710 & $0.52150^{1,2,3}$ & 1 & 2.4200 & 5.470 & 6.790 & 9.730 & $0.44840^{1,2,3}$ \\
\hline Seychelles & 0 & 8.1700 & 13.880 & 15.760 & 19.710 & $0.52740^{1,2,3}$ & 1 & 0.6100 & 5.470 & 6.790 & 9.730 & $0.89520^{1,2,3}$ \\
\hline Sierra Leone & 0 & 8.5500 & 13.880 & 15.760 & 19.710 & $0.48560^{1,2,3}$ & 1 & 1.6100 & 5.470 & 6.790 & 9.730 & $0.63200^{1,2,3}$ \\
\hline South Africa & 0 & 7.9700 & 13.880 & 15.760 & 19.710 & $0.55080^{1,2,3}$ & 1 & 0.4700 & 5.470 & 6.790 & 9.730 & $0.92660^{2,3}$ \\
\hline Sudan & 0 & 6.3500 & 13.880 & 15.760 & 19.710 & $0.73700^{1,2,3}$ & 1 & 0.2800 & 5.470 & 6.790 & 9.730 & $0.96690^{3}$ \\
\hline Swaziland & 0 & 8.8100 & 13.880 & 15.760 & 19.710 & $0.45690^{1,2,3}$ & 1 & 0.5900 & 5.470 & 6.790 & 9.730 & $0.89900^{1,2,3}$ \\
\hline Togo & 0 & 8.0500 & 13.880 & 15.760 & 19.710 & $0.54180^{1,2,3}$ & 1 & 0.5100 & 5.470 & 6.790 & 9.730 & $0.91750^{2,3}$ \\
\hline Uganda & 0 & 14.9200 & 13.880 & 15.760 & 19.710 & $0.06860^{1,2,3}$ & 1 & 0.6000 & 5.470 & 6.790 & 9.730 & $0.89650^{1,2,3}$ \\
\hline Zambia & 0 & 7.0000 & 13.880 & 15.760 & 19.710 & $0.66270^{1,2,3}$ & 1 & 0.6900 & 5.470 & 6.790 & 9.730 & $0.87340^{1,2,3}$ \\
\hline Zimbabwe & 0 & 9.9500 & 13.880 & 15.760 & 19.710 & $0.34300^{1,2,3}$ & 1 & 0.1300 & 5.470 & 6.790 & 9.730 & $0.98990^{3}$ \\
\hline Bangladesh & 0 & 19.9800 & 13.880 & 15.760 & 19.710 & $0.00890^{1,2,3}$ & 1 & 0.5400 & 5.470 & 6.790 & 9.730 & $0.91110^{2,3}$ \\
\hline Hong Kong & 0 & 7.2300 & 13.880 & 15.760 & 19.710 & $0.63670^{1,2,3}$ & 1 & 1.7200 & 5.470 & 6.790 & 9.730 & $0.60530^{1,2,3}$ \\
\hline India & 0 & 6.0100 & 13.880 & 15.760 & 19.710 & $0.77370^{1,2,3}$ & 1 & 0.4900 & 5.470 & 6.790 & 9.730 & $0.92320^{2,3}$ \\
\hline Israel & 0 & 6.5400 & 13.880 & 15.760 & 19.710 & $0.71520^{1,2,3}$ & 1 & 0.4300 & 5.470 & 6.790 & 9.730 & $0.93640^{2,3}$ \\
\hline Japan & 0 & 8.5700 & 13.880 & 15.760 & 19.710 & $0.48340^{1,2,3}$ & 1 & 0.4600 & 5.470 & 6.790 & 9.730 & $0.92920^{2,3}$ \\
\hline Malaysia & 0 & 5.3800 & 13.880 & 15.760 & 19.710 & $0.83610^{1,2,3}$ & 1 & 0.6600 & 5.470 & 6.790 & 9.730 & $0.88220^{1,2,3}$ \\
\hline Nepal & 0 & 13.3600 & 13.880 & 15.760 & 19.710 & $0.11990^{1,2,3}$ & 1 & 0.8800 & 5.470 & 6.790 & 9.730 & $0.82530^{1,2,3}$ \\
\hline Oman & 0 & 6.5800 & 13.880 & 15.760 & 19.710 & $0.71050^{1,2,3}$ & 1 & 0.0300 & 5.470 & 6.790 & 9.730 & $0.99890^{3}$ \\
\hline Pakistan & 0 & 9.9100 & 13.880 & 15.760 & 19.710 & $0.34630^{1,2,3}$ & 1 & 0.5500 & 5.470 & 6.790 & 9.730 & $0.90890^{2,3}$ \\
\hline
\end{tabular}

Note: ${ }^{1}$ shows statistical significance at $90 \%$ critical level; ${ }^{2}$ shows statistical significance at $95 \%$ critical level; ${ }^{3}$ shows statistical significance at $99 \%$ critical level. Note that $\rho$-values less than critical levels of $90 \%, 95 \%$ and $99 \%$ represent cointegration. The test was carried out using JMulti 4 statistical package. The deterministic term of the VECM was defined as $D_{t}=u_{o}+u_{1 t}$. Superscripts 1, 2, 3 show statistical significance at 90\%, 95\%, and 99\% critical levels. LR $=$ Likelihood Ratio.

Eviews 7 was used to carry out the Toda and Yamamoto (1995) approach to causality. The results show that Brazil's income is induced by China, South Africa and India's economic growth. The countries registered $\rho$-values less than the $5 \%$ critical level suggesting that we have to reject the null hypothesis of non-causality. Table 4 is a presentation of the VAR estimates before the causality test. Table 5 presents the results of the Toda and Yamamoto (1995) causality test. 
Table 4: Vector Autoregression (VAR) Estimates

\begin{tabular}{|c|c|c|c|c|}
\hline & BRAZIL & INDIA & CHINA & SOUTH AFRICA \\
\hline BRAZIL_LN(-1) & $\begin{array}{l}0.893856 \\
(0.14585) \\
{[6.12849]}\end{array}$ & $\begin{array}{l}0.159168 \\
(0.10593) \\
{[1.50255]}\end{array}$ & $\begin{array}{c}-0.053817 \\
(0.10323) \\
{[-0.52131]}\end{array}$ & $\begin{array}{c}0.09826 \\
(0.14993) \\
{[0.65779]}\end{array}$ \\
\hline BRAZIL_LN(-2) & $\begin{array}{l}-0.251374 \\
(0.13123) \\
{[-1.91556]}\end{array}$ & $\begin{array}{c}-0.139890 \\
(0.09531) \\
{[-1.46774]}\end{array}$ & $\begin{array}{c}-0.058306 \\
(0.09288) \\
{[-0.62774]}\end{array}$ & $\begin{array}{l}0.020327 \\
(0.13490) \\
{[0.15068]}\end{array}$ \\
\hline INDIA_LN(-1) & $\begin{array}{c}0.146953 \\
(0.234970) \\
{[0.625420]}\end{array}$ & $\begin{array}{c}0.857351 \\
(0.170650) \\
{[5.02391]}\end{array}$ & $\begin{array}{c}0.257046 \\
(0.166310) \\
{[1.545600]}\end{array}$ & $\begin{array}{c}0.262708 \\
(0.241540) \\
{[1.087630]}\end{array}$ \\
\hline INDIA_LN(-2) & $\begin{array}{c}-0.555782 \\
(0.244730) \\
{[-2.270990]}\end{array}$ & $\begin{array}{c}-0.050748 \\
(0.177750) \\
{[-0.285500]}\end{array}$ & $\begin{array}{c}0.283923 \\
(0.173220) \\
{[-1.639080]}\end{array}$ & $\begin{array}{c}-0.126563 \\
(0.251580) \\
{[-0.503070]}\end{array}$ \\
\hline CHINA_LN(-1) & $\begin{array}{c}0.155202 \\
(0.199810) \\
{[0.776750]}\end{array}$ & $\begin{array}{c}-0.077558 \\
(0.145120) \\
{[-0.534440]}\end{array}$ & $\begin{array}{c}1.090469 \\
(0.141430) \\
{[7.710570]}\end{array}$ & $\begin{array}{c}-0.073913 \\
(0.205400) \\
{[-0.359840]}\end{array}$ \\
\hline CHINA_LN(-2) & $\begin{array}{c}0.0838210 \\
(0.2085500) \\
{[0.401920}\end{array}$ & $\begin{array}{c}0.1885270 \\
0.151470 \\
{[1.244660]}\end{array}$ & $\begin{array}{c}-0.0493370 \\
0.147610 \\
{[-0.334230]}\end{array}$ & $\begin{array}{c}0.030952 \\
0.214390 \\
{[0.144370]}\end{array}$ \\
\hline SA_LN(-1) & $\begin{array}{l}0.4900440 \\
(0.174350) \\
{[2.810750]}\end{array}$ & $\begin{array}{c}0.093754 \\
(0.126630) \\
{[0.740400]}\end{array}$ & $\begin{array}{l}0.0298370 \\
(0.123400) \\
{[0.241780]}\end{array}$ & $\begin{array}{c}1.066611 \\
(0.179230) \\
{[5.951210]}\end{array}$ \\
\hline SA_LN(-2) & $\begin{array}{c}0.030164 \\
(0.183150) \\
{[0.164700]}\end{array}$ & $\begin{array}{c}-0.055641 \\
(0.133020) \\
{[-0.418300]}\end{array}$ & $\begin{array}{c}0.1072180 \\
(0.129630) \\
{[0.827100]}\end{array}$ & $\begin{array}{c}-0.313102 \\
(0.188270) \\
{[-1.663030]}\end{array}$ \\
\hline $\mathrm{C}$ & $\begin{array}{c}0.602748 \\
(0.211400) \\
{[2.851220]}\end{array}$ & $\begin{array}{c}0.1964430 \\
(0.153540) \\
{[1.279440]}\end{array}$ & $\begin{array}{l}0.0129480 \\
(0.149630) \\
{[0.086530]}\end{array}$ & $\begin{array}{c}-0.049159 \\
(0.217320) \\
{[-0.226210]}\end{array}$ \\
\hline
\end{tabular}


Table 5: Toda and Yamamoto Causality Test Results

\begin{tabular}{|c|c|c|c|c|}
\hline \multicolumn{5}{|c|}{ Dependent: Brazil } \\
\hline Country & Chi-square & $d f$ & $\rho$-value & Causation \\
\hline India & 7.336837 & 2 & $0.02550^{*}$ & India $\Rightarrow$ Brazil \\
\hline China & 7.727293 & 2 & $0.02100 *$ & China $\Rightarrow$ Brazil \\
\hline South Africa & 14.40729 & 2 & $0.00070^{*}$ & $\mathrm{SA} \Rightarrow$ Brazil \\
\hline \multicolumn{5}{|c|}{ Dependent: India } \\
\hline Country & Chi-square & $d f$ & $\rho$-value & Causation \\
\hline Brazil & 2.520325 & 2 & 0.28360 & Brazil $\nRightarrow$ India \\
\hline China & 3.621016 & 2 & 0.16360 & China $\nRightarrow$ India \\
\hline South Africa & 0.553305 & 2 & 0.75830 & $\mathrm{SA} \Leftrightarrow$ India \\
\hline \multicolumn{5}{|c|}{ Dependent: China } \\
\hline Country & Chi-square & $d f$ & $\rho$-value & Causation \\
\hline Brazil & 2.665138 & 2 & 0.26380 & Brazil $\nRightarrow$ China \\
\hline India & 2.957489 & 2 & 0.22790 & India $\Leftrightarrow$ China \\
\hline South Africa & 1.688156 & 2 & 0.43000 & $\mathrm{SA} \nRightarrow$ China \\
\hline \multicolumn{5}{|c|}{ Dependent: South Africa } \\
\hline Country & Chi-square & $d f$ & $\rho$-value & Causation \\
\hline Brazil & 1.391189 & 2 & 0.49880 & Brazil $\Leftrightarrow S A$ \\
\hline India & 1.352688 & 2 & 0.50850 & India $\Leftrightarrow S A$ \\
\hline China & 0.323568 & 2 & 0.85060 & China $\nRightarrow S A$ \\
\hline
\end{tabular}

Note: The arrows signify the direction of causation. $\Rightarrow$ implies causality in a given direction; $\nRightarrow$ implies that there is no causality between the variables. The test was carried out at 5\% significant level. The null hypothesis $\left(\mathrm{H}_{0}\right)$ is that a given variable does not Granger cause the other (non-causality). Note that $\rho$-values less than the $5 \%$ critical level $(\rho<0.05)$ represent causality in a given direction. The null hypothesis is therefore rejected for $\rho$-values less than the significant level. Asterisks $\left(^{*}\right)$ represent a causal relationship at the 5\% significant level. Eviews (7) was used to carry out the Toda-Yamamoto approach to Granger causality.

\section{Discussion and Conclusion}

This study aimed to determine the influence the BRICS have on other economies economic growth. The other objective of this empirical investigation was to determine the causal relations between the BRICS's economic growth. Economic growth exhibited by the BRICS has been impressive in the last decade. China is currently the largest economy after the US but her economic growth has been attached with shortcomings. Economic growth is every country's major goal however there are environmental costs. The BRICS's economic growth has been attributed to exports variety and industrialisation. This paper aimed to find out if such rapid growth could potentially affect economic growth of other nations. In this investigation the Saikkonen and Lütkepohl (2000) cointegration model has been applied to validate long term relationships between economic growth series. The Toda and Yamamoto (1995) approach to Granger causality was applied to investigate the influence the BRICS have on each other's economic growth over the period 1980 to 2013. The results of the empirical analysis show that all countries' depict long term relationships between China and Brazil's economic growth 
pattern. In addition, the causality test shows that South Africa, India and China induce Brazil's economic growth.

The results of this study carry implications for future world economic growth. All economies under investigation revealed long term relations with China and Brazil's economic growth. To a reasonable extent, this shows a state of dependency between the BRICS and such nations. China and Brazil need to export their goods and services to developing nations. Developing economies also require such goods and services for their own economic development and industrialisation attempts. Brazil's economic growth is induced by other BRICS members namely South Africa, China and India. This suggests that economic and financial integration is required among the BRICS. If these economies heighten economic and financial cooperation, other developing economies will also benefit from this collaboration. Even though the BRICS are competing in terms of exports sales, economic and financial is still a necessity for high economic growth. In conclusion of this study, the BRICS are influential in world economic growth. However, their economic prosperity is also dependent on integration.

\section{References}

Amador, J. (2012). 'Energy content in manufacturing exports: A cross country analysis'. Energy Economics, Vol. 34, pp. 1074:1081.

Chang, Chun-Ping, Na Berdiev, A. and Lee, Chien-Chiang (2013). 'Energy exports, globalization and economic growth: The case of South Caucasus'. Economic Modelling, Vol. 33, pp. 383-346.

Sharma, K. (2003). 'Factors determining India's export performance'. Journal of Asian Economics, Vol. 14, pp. 435-446.

Tekin, R. B. (2012). 'Economic growth, exports and foreign direct investment in least developed countries: a panel Granger causality analysis'. Economic Modelling, 29: 868-878.

Asemota, O. M., and Bala, D. A. (2011). 'A Kalman Filter approach to Fisher effect: Evidence from Nigeria’. CBN Journal of Applied Statistics, Vol. 2, No. 1, pp. 71-91.

Caporale, G. M., and Pittis, N. (1999). 'Efficient estimation of cointegrating vectors and testing for causality in vector autoregressions'. Journal of Economic Issues, Vol. 13, pp. 3-35.

Chang, Chun-Ping., Berdiev, A. N., and Lee, Chien-Chiang. (2013) 'Energy exports, globalization and economic growth: The case of South Caucasus'. Economic Modelling, Vol. 33, pp.383-346.

Dickey, D. A., and Fuller, W. A. (1979). 'Distribution of the estimators for autoregressive time series with a unit root'. Journal of the American Statistical Association, Vol. 74, No. 366, pp. 427-431.

Elliott, G. (1998). 'On the robustness of cointegration methods when regressors almost have a unit root'. Econometrica, Vol. 66, No.1, pp. 149-158. doi:10.2307/2998544.

Engle, R. F., and Granger, C. W. (1987). 'Cointegration and error correction: representation, estimation, and testing'. Econometrica, Vol. 55, No. 2, pp. 251-276. doi:10.2307/1913236. 
Granger, C. J. (1981). 'Some properties of time series data and their use in econometric model specification'. Journal of Econometrics, Vol. 55, pp. 251-276. doi:10.1016/03044076(81)90079-8.

Granger, C. W. J. and Engle, R. F. (1985). 'Dynamic model specification with equilibrium constraints'. University of California, San Diego.

Granger, C. W. J., and Weiss, A. A. (1983). 'Time series analysis of error correction models'. In Karlim, S; Amemiya, T., and Goodman, L. A. Studies in Economic Time Series and Multivariate Statistics, (Academic Press: New York). doi:10.1016/b978-0-12-3987501.50018-8.

Granger, C. W. J. (1969). 'Investigating causal relations by econometric models: cross spectral methods'. Econometrica, Vol. 37, No. 3, pp. 424- 438. doi:10.2307/1912791.

http://www.theglobaleconomy.com/ (Retrieved 11 January 2016).

Hovarth, W., and Watson, M. (1995). 'Testing for cointegration when some of the cointegrating vectors are prespecified'. Econometric Theory, Vol. 11, No.5, pp. 984-1014. doi:10.1017/s0266466600009944.

Johansen, S. (1991a). 'Estimation and hypothesis testing of cointegration vectors in Gaussian Vector Autoregressive models'. Econometrica, Vol. 59, No. 6, pp. 1551-1580. doi: $10.2307 / 2938278$.

Johansen, S. (1988b). 'Statistical analysis of cointegration vectors'. Journal of Economic Dynamics and Control, Vol. 12, pp. 231-254. doi:10.1016/0165-1889(88)90041-3.

Johansen, S., and Juselius, K. (1990). 'Maximum likelihood estimation and inference on cointegration with applications to the demand for money'. Oxford Bulletin of Economics and Statistics, Vol. 52, No. 2, pp. 169-210. doi:10.1111/j.1468-0084.1990.mp52002003.x.

Mavrotas, G., and Kelly, R. (2001). 'Old wine in new bottle: testing causality between savings and growth’. The Manchester School Supplement, pp. 97-105.

Park, J. Y. (1992a). 'Canonical cointegration regression'. Econometrica, Vol. 60, pp.119-144.

Park, J. Y. (1990b). 'Testing for unit roots and cointegration by variable addition'. Advances in Econometrics, Vol. 8, pp. 107-133.

Phillips, P. C. B and Durlauf, S. N. (1986). 'Multiple time series regression with integrated processes'. Review of Economic Studies, Vol. 53, pp. 473-495. doi:10.2307/2297602.

Phillips, P. C. B., and Hansen, B. E (1990). 'Statistical inference in instrumental variable with I(1) processes'. Review of Economic Studies, Vol. 57, pp. 99-125. doi:10.2307/2297545.

Phillips, P. C. B., and Ouilaris, S. (1986). 'Testing for cointegration'. Cowles Foundation Discussion Paper, No. 809.

Phillips, P. C. B., and Park, J. Y. (1986). 'Asymptotic equivalence of OLS and GLS in regression with integrated regressors'. Cowles Foundation Discussion Paper, No. 802.

Phillips, P. C. B., and Perron, P. (1988). 'Testing for a unit root in time series regression'. Biometrika, Vol. 75, No. 2, pp. 335-346. doi:10.2307/2336182. 
Rambaldi, A. N. (1997). 'Mutliple time series models and testing for causality and exogeneity: a review'. Working Papers in Econometrics and Applied Statistics, No. 96. Department of Econometrics, University of New England.

Rambaldi, A. N., and Doran, H. E. (1996). 'Testing for Granger non-causality in cointegrated systems made easy'. Working Papers in Econometrics and Applied Statistics, No.88. Department of Econometrics, University of New England.

Saikkonen, P. (1992). 'Estimation and testing of cointegrated systems by an autoregressive approximation'. Econometric Theory, Vol. 8 No. 1, pp. 1-27. doi:10.1017/s0266466600010720.

Saikkonen, P., and Lütkepohl, H. (2000). 'Testing for the cointegrating rank of a VAR process with an intercept'. Economic Theory, Vol. 16, pp. 373-406.

Stock, J. H. (1987). 'Asymptotic properties of least squares estimates of cointegration vectors'. Econometrica, Vol. 55, No. 5, pp. 1035-1056. doi:10.2307/1911260.

Stock, J. H., and Watson, M. W (1987). 'Testing for common trends'. Working Paper in Econometrics (Hoover Institution, Stanford, CA).

Toda., H. Y., and Yamamoto, T. (1995). 'Statistical inference in vector autoregressions with possibly integrated processes'. Journal of Econometrics, Vol. 66, pp. 225-250.

Wolde-Rufael, Y. (2005). 'Energy demand and economic growth: the African experience'. Journal of Policy Modeling, Vol. 27, pp. 891-903. doi:10.1016/j.jpolmod.2005.06.003.

Zapata, H, O., and Rambaldi, A. N. (1997). 'Monte Carlo evidence on cointegration and causation'. Oxford Bulletin of Economics and Statistics, Vol. 59, pp. 285-298. 